\title{
ASSOCIATIONS BETWEEN VASPIN RS2236242 GENE POLYMORPHISM, WALKING TIME AND THE RISK OF METABOLIC SYNDROME
}

\author{
Suliga $E^{1}$, Kozieł $D^{2}$, Cieśla $E^{3}$, Rę̧bak $D^{2}$, Wawszczak M ${ }^{2 *}$, \\ Adamus-Białek $W^{2}$, Naszydłowska $E^{4}$, Piechowska $A^{2}$, Głuszek $\mathbf{S}^{2}$
}

*Corresponding Author: Monika Wawszczak, MSc, Department of Surgery and Surgical Nursery with Genetics and Research Laboratory, Institute of Medical Sciences, Jan Kochanowski University, al. IX Wieków Kielc 19a, 25-516, Kielce, Poland. Tel: +48-413696978. E-mail: mwawszczak@ujk.edu.pl

\begin{abstract}
The associations between serum vaspin levels and metabolic or coronary artery disease (CAD) and polycystic ovary syndrome (PCOS) is under the scope of current researchers. Therefore, this adipokine can be considered as a biomarker of metabolic syndrome (MetS). The aim of the study was to analyze the associations between the vaspin rs2236242 polymorphism and physical activity in relation to MetS and its components. The analysis involved the genetic material and clinical data of 108 individuals with MetS and 110 controls. Vaspin rs2236242 polymorphism was detected using the tetra-primer amplification-refractory mutation system polymerase chain reaction (T-ARMS PCR) method. The TA genotype of vaspin rs2236242 was associated with a greater risk of MetS and its components compared with the TT genotype. The analysis of interactions between genotype and walking time revealed that a walking time longer than $60 \mathrm{~min}$./day significantly decreased the risk of MetS in the TA carriers $(p=0.007)$. The obtained results suggest that any unfavorable effect of the TA genotype of the vaspin rs 2236242 polymorphism can be essentially reduced, or even reversed, in a case of indi-

\footnotetext{
${ }^{1}$ Department of Nutrition and Dietetics, Institute of Public Health, Faculty of Medicine and Health Sciences, Jan Kochanowski University, Kielce, Poland Laboratory and Research Laboratory, Institute of Medical Sciences, Jan Kochanowski University, Kielce, Poland Health, Faculty of Medicine and Health Sciences, Jan Kochanowsk University, Kielce, Poland Medicine and Health Sciences, Jan Kochanowski University, Kielce, Poland
}

${ }^{2}$ Department of Surgery and Surgical Nursery with Genetics

${ }^{3}$ Department of Developmental Age Research, Institute of Public

${ }^{4}$ Department of Social Prevention, Institute of Public Health, Faculty of
\end{abstract}

viduals walking longer than $60 \mathrm{~min}$. a day. The analysis of the interaction between vaspin rs 2236242 polymorphism and walking showed that a walking time of longer than 1 hour a day significantly reduced the risk of MetS, elevated blood pressure and triglycerides concentration.

Keywords: Abdominal; Adipokines; Exercise; Metabolic syndrome (MetS); Obesity.

\section{INTRODUCTION}

Vaspin (visceral adipose tissue-derived serine protease inhibitor) was isolated for the first time from visceral adipose tissue in Otsuka Long-Evans Tokushima Fatty rats, which comprises an animal model of abdominal obesity, accompanied by type 2 diabetes mellitus (T2DM) [1]. Human vaspin mRNA has been found both in the visceral and in subcutaneous adipose tissue. It is coded by the gene SERPNA12 located on the long arm of chromosome 14 (14q32.13) and consists of five introns and six exons. According to the literature review, there is a positive association between the vaspin concentration in the blood serum and the indicators of obesity, T2DM, polycystic ovary syndrome (PCOS) and coronary artery disease (CAD) [1,2]. Feng et al. [2] after the meta-analysis of the scores of six studies involving 1826 participants, revealed that the vaspin levels were aproximately $0.52 \mathrm{ng} / \mathrm{mL}$ higher in obese patients than in controls. However, some studies showed a lack of associations between the level of vaspin and obesity [3,4]. Several researchers suggest that this adipokin increases the insulin sensitivity of adipose tissue in the condition of obesity. The increase of vaspin expression acts like a compensatory mechanism and being a reaction to growing obesity and insulin resistance. Several studies have revealed that vaspin concentration 
is related to a better metabolic profile and that its levels in the blood serum are significantly higher in individuals with metabolic syndrome (MetS) than in control $[5,6]$. So far, only a few studies devoted to the analysis of the relationships between vaspin gene polymorphism and the risk of MetS have been published [6-8]. Studies conducted by Hashemi et al. [7] and Mehanna et al. [8] confirmed that the risk of MetS was significantly lower in allele A rs2236242 carriers. On the other hand, Alnory et al. [6] in the studies involving the Egyptian population, did not find any differences between the frequency of vaspin rs 2236242 genotypes and the risk of MetS. Therefore, it was stated that there are only a few studies concerning association between vaspin gene polymorphism and MetS, and their results are ambiguous. No such study has been conducted in the Polish population. According to our current knowledge, none of the previously published studies considered relationship between vaspin rs2236242 polymorphism and the risk of MetS including physical activity or walking. Therefore, the aim of the study was the analysis of the associations between vaspin rs 2236242 polymorphism and physical activity in relation to MetS and its components.

\section{MATERIALS AND METHODS}

Study Population. Genomic DNA isolated from 108 participants with MetS and 110 controls of the PolishNorwegian Study (PONS) project were used in this study. All participants were Caucasians. The study included blood pressure measurements, the analysis of collected fasting-blood samples, anthropometric measurements and a questionnaire interview. Detailed information regarding the project and research procedures were described in a previously published study [9] In 108 participants, MetS was diagnosed on the basis of the International Diabetes Federation criteria [10].

Ethics. The study was approved by the Ethics Committee from the Cancer Centre and Institute of Oncology in Warsaw (data collection) and by the Committee on Bioethics at the Faculty of Health Sciences, Jan Kochanowski University in Kielce, Poland (data analysis) (decision number: 45/2016).

Genotyping. Determination of the vaspin rs2236242 polymorphism was conducted by the tetra-primer amplification-refractory mutation system polymerase chain reaction (T-ARMS PCR) method, according to the protocol described by Hashemi et al. [7]. The external primers VasFO (5'-GGA GGC AGA CCA GGC ACT AGA AA-3') and VasRO (5'-ACC ATC TCT CTG GCT TCA GGC TTC3') were applied for the amplification of $378 \mathrm{bp}$ DNA of vaspin gene fragment. Inter primers were specific to each genotype: Vas FI primer (5'-AAG ACG CCG CTT CTG
TGC ACT-3') for the T allele and Vas RI primer (5'-CAC AGG GAC CCA GGA TAA CTT GCT3') for the A allele.

The commercially available PCR premix (PCR Mix Plus; A\&A Biotechnology, Gdynia, Poland) was prepared according to the manufacturer's instructions. Briefly, $1 \mu \mathrm{L}$ of each primer $(10 \mathrm{pmol} / \mu \mathrm{L}), 1 \mu \mathrm{L}$ of template DNA $(\sim 25$ $\mathrm{ng} / \mu \mathrm{L}), 12.5 \mu \mathrm{L}$ of PCR Pre Mix and made up to $25 \mu \mathrm{L}$ with DNAse-free water. The procedure of T-ARMS PCR was optimized and final conditions were: denaturation at $95^{\circ} \mathrm{C}$ for $3 \mathrm{~min}$., amplification for 30 cycles at $95^{\circ} \mathrm{C}$ for $1 \mathrm{~min}$., $60^{\circ} \mathrm{C}$ for $1 \mathrm{~min}$., and $72{ }^{\circ} \mathrm{C}$ for $1 \mathrm{~min}$., and a final extension at $72{ }^{\circ} \mathrm{C}$ for $8 \mathrm{~min}$. After DNA amplification, the gel documentation system (InGenius; Syngene, Cambridge, Cambridgeshire, UK) was used for product visualization. The specific genotypes were characterized, respectively: 174 and 378 bp for homozygous T, 248 and $378 \mathrm{bp}$ for homozygous $\mathrm{A}$, and 174, 248 and $378 \mathrm{bp}$ for heterozygous TA (Figure 1).

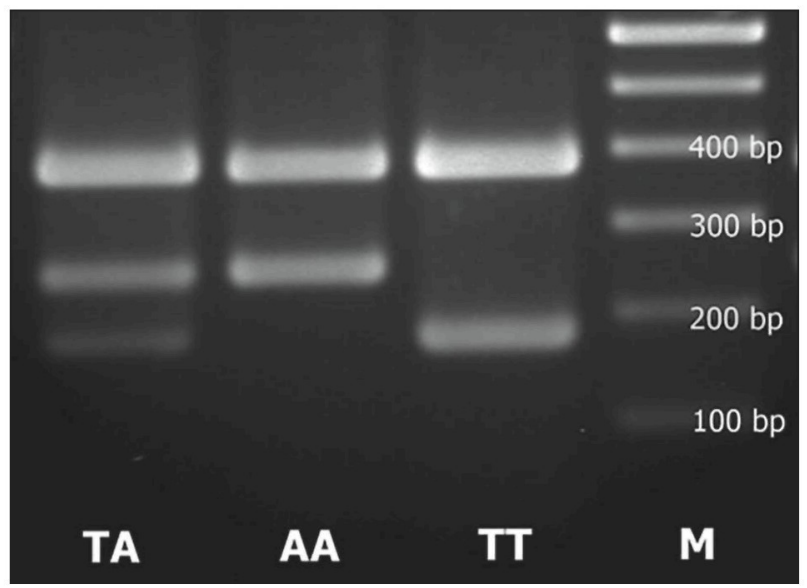

Figure 1. Electrophoresis patterns of T-ARMS PCR for the detection of SNPs in vaspin rs2236242. The product sizes were $174 \mathrm{bp}$ for T allele, $248 \mathrm{bp}$ for A allele, and $378 \mathrm{bp}$ for control. $\mathrm{M}$; DNA marker.

Meta-Analysis of the Vaspin Genotypes. The metaanalysis of the vaspin rs2236242 polymorphism included the data from Iran [7], Egypt [6,11] and Poland (this study). The distribution of the genotype was examined in MetS volunteers $(n=459)$ and controls $(n=459)$.

Statistical Analyses. All data were analyzed using the Statistical Package Statistica software (version 13.1) (TIBCO Software Inc., Palo Alto, CA, USA). Mean \pm standard deviation (SD) and medians with interquartile ranges (Me; Q1-Q3) for continuous variables and proportions expressed as percentages for categorical variables, were used to describe the baseline characteristic of the group. Differences between MetS participants and the control group were assessed through the $\chi^{2}$ test and the $\mathrm{U}$ Mann-Whitney-test. The $\chi^{2}$ test was also used to analyze 
genotype and allele frequencies and to determine whether the genotype distribution was congruent with Hardy-Weinberg equilibrium expectations. The multivariate logistic regression analyses were used to estimate the odds ratio (OR) and $95 \%$ confidence interval ( $95 \% \mathrm{CI}$ ) for MetS and its five components. The TT was a reference genotype in the analysis. In the second model, the risk for MetS and its components was adjusted for age, gender, walking time $(\leq$ or $>60 \mathrm{~min}$./day) and smoking. The choice of confounders in models II and III was based on the scores obtained from the cluster analysis. It was performed on raw values by means of a single linkage method. A distance measurement was the Euclidean distance. In the third model, body mass index (BMI), was added (as a continuous variable). Additionally, in models II and III, interactions between vaspin rs2236242 gene polymorphism and walking, in relation to MetS and its components, were considered. A reference point for walking was time $\leq 60 \mathrm{~min}$./day. In all analyses, a $p$ value of $<0.05$ was considered to be statistically significant.

\section{RESULTS}

The study involved 108 individuals with MetS and 110 participants representing the control group. The group with MetS was similar to the control group regarding sex and age. Participants with MetS were more often overweight (BMI $>25.0 \mathrm{~m} / \mathrm{kg}^{2}$ ), with abdominal obesity, elevated blood pressure, glucose and triglyceride (TG) concentration and decreased concentration of high density lipoprotein (HDL) cholesterol, compared to the control group (all $p<0.001$ ). No significant differences were found between the percentages of current and former smokers in both groups $(p=0.456)$. However, the individuals with MetS were characterized by a significantly shorter time of a total physical activity (PA) during the day (232.2 \pm $144.4 \mathrm{~min} . /$ day $v s .270 .6 \pm 145.6 \mathrm{~min} . /$ day; $p=0.039$ ), including mainly, a shorter walking time $(79.6 \pm 71.1 \mathrm{~min} . /$ day $v s$. $109.2 \pm 85.5 \mathrm{~min} . / \mathrm{day} ; p=0.007)$, compared to the control group.

There was no statistically significant difference in distribution of alleles and genotypes of the vaspin rs2236242 polymorphism between individuals with MetS and controls (Table 1). The meta-analysis of genotype distribution in Egyptian, Iranian and Polish populations showed that the TT genotype was identified more frequently in MetS patients than in controls $(p=0.001)$. The A allele occurrence was higher in the control group ( $p=0.001)$ (Table 2).

However, a two-fold more frequent prevalence of the AA genotype in individuals from the control group compared to the group with MetS was found (15.45 vs. $\mathbf{8 . 3 3 \%}$ ). There was also a tendency to a slightly more frequent prevalence of the TA genotype in individuals with MetS, compared to the control group (51.86 vs. $45.45 \%$ ). The genotype distribution showed no deviation from the Hardy-Weinberg equilibrium in the whole study sam-

Table 1. Genotype and allele distribution of the vaspin rs2236242 polymorphism in the control group and MetS patients.

\begin{tabular}{|c|c|c|c|c|c|}
\hline Genotypes & $\begin{array}{l}\text { Total } \\
n(\%)\end{array}$ & $\begin{array}{c}\text { Control Group } \\
n(\%)\end{array}$ & $\begin{array}{c}\text { MetS Patients } \\
n(\%)\end{array}$ & $\chi^{2}(\mathbf{d f}=2)$ & $p$ Value \\
\hline TT & $86(39.45)$ & $43(39.10)$ & $43(39.81)$ & & \\
\hline $\mathrm{TA}$ & $106(48.62)$ & $50(45.45)$ & $56(51.86)$ & 2.783 & 0.250 \\
\hline AA & $26(11.93)$ & $17(15.45)$ & $9(8.33)$ & & \\
\hline Alleles & & & & $\chi^{2}(\mathrm{df}=1)$ & $p$ Value \\
\hline $\mathrm{T}$ & $278(63.76)$ & $126(61.82)$ & $142(65.74)$ & 0.730 & 0.394 \\
\hline $\mathrm{A}$ & $158(36.24)$ & $84(38.18)$ & $74(34.26)$ & & \\
\hline
\end{tabular}

MetS: metabolic syndrome.

Table 2. The meta-analysis of the the vaspin rs2236242 polymorphism in the MetS patients $(n=469)$ and the control group $(n=459)$ from Iran, Egypt and Poland.

\begin{tabular}{|c|c|c|c|c|}
\hline Genotypes & $\begin{array}{c}\text { MetS Patients } \\
\boldsymbol{n}(\%)\end{array}$ & $\begin{array}{c}\text { Control Group } \\
\boldsymbol{n}(\%)\end{array}$ & $\boldsymbol{\chi}^{\mathbf{2}(\mathbf{d f}=\mathbf{2})}$ & $\boldsymbol{p}$ Value \\
\hline TT & $210(44.78)$ & $149(32.46)$ & & $<0.0001$ \\
\hline TA & $215(45.84)$ & $240(52.59)$ & 24.20 & $p$ Value \\
\hline AA & $34(7.25)$ & $70(15.25)$ & & $<0.0001$ \\
\hline Alleles & & & $\chi^{2}(\mathrm{df}=1)$ & 33.21 \\
\hline T & $635(67.70)$ & $538(58.61)$ & & \\
\hline A & $283(30.17)$ & $380(41.39)$ & & \\
\hline
\end{tabular}

MetS: metabolic syndrome. 
ple $\left(\chi^{2}=0.536 ; p=0.464\right)$, in subjects with $\operatorname{MetS}\left(\chi^{2}=\right.$ $2.467 ; p=0.116)$, and in the control group $\left(\chi^{2}=0.200 ; p\right.$ $=0.655)$. The A allele frequency of the vaspin rs 2236242 polymorphism was 0.360 in total, 0.343 in MetS and 0.378 in the control group.

The TA, AA and TA+AA genotypes showed no association with MetS when compared to the TT genotype ( $p=0.638 ; p=0.187 ; p=0.979$, respectively). The results of the conducted study also revealed that the vaspin rs2236242 polymorphism was not significantly associated with any of the MetS components. Despite a lack of statistically significant associations, having the AA genotype (compared to TT) was related to quite a clear tendency to a lower risk of MetS and its components (OR varied from 0.54 to 0.80 ) (Table 3 , model I).

In the model adjusted for age, gender, smoking and total PA, no statistically significant correlations have been found between the vaspin rs2236242 gene polymorphism and the risk of MetS and its components (data not shown). The differences of PA between MetS-subjects and the control group resulted mainly from the differences of walking time. The group of variables of small linkage distances included all MetS components, some demographic variables and lifestyle elements, along with walking time (Figure 2). Total PA and moderate-to-vigorous PA were characterized by greater Euclidean distances than the others. Taking this fact into consideration, in subsequent models walking time instead of total PA was used as a modifying variable. In the model adjusted for age, gender, smoking and walking (model II), there were also no significant associations between the vaspin rs 2236242 polymorphism and MetS components. However, it was noted that having the TA genotype was related to a greater risk of MetS, compared to the TT genotype. The analysis of interaction 'genotype $\times$ walking time' showed that a longer walking time $(>60$ $\mathrm{min} . /$ day) statistically significantly decreased the risk of

Table 3. Associations between the vaspin rs2236242 polymorphism a risk of MetS and its components [OR (95\% CI)]. I: unadjusted model; II: model adjusted for gender, age, smoking, walking and interactions between the vaspin rs 2236242 polymorphism and walking in relation to MetS and its components; III: model adjusted for gender, age, smoking, BMI, walking and interactions between the vaspin rs2236242 gene polymorphism and walking in relation to MetS or its components.

\begin{tabular}{|c|c|c|c|c|c|c|c|c|c|}
\hline \multicolumn{2}{|l|}{ Components of MetS } & $\begin{array}{c}\text { AA } \\
\text { (TT ref.) }\end{array}$ & $p$ Value & $\begin{array}{c}\text { TA } \\
\text { (TT ref) } \\
\end{array}$ & $p$ Value & $\begin{array}{r}\text { TA+AA } \\
\text { (TT ref.) } \\
\end{array}$ & $p$ Value & $\begin{array}{c}\mathbf{A A} \\
\text { (TT+TA ref.) }\end{array}$ & $p$ Value \\
\hline \multirow[t]{3}{*}{ MetS } & I & $0.54(0.22-1.35)$ & 0.187 & $0.15(0.65-2.02)$ & 0.638 & $0.99(0.58-1.71)$ & 0.979 & $0.50(0.21-1.18)$ & 0.115 \\
\hline & II & $1.24(0.31-4.97)$ & 0.759 & $2.54(1.07-60.5)^{a}$ & 0.035 & $2.14(0.96-4.77)^{\mathrm{a}}$ & 0.064 & $0.75(0.20-2.83)$ & 0.674 \\
\hline & III & $0.91(0.19-4.36$ & 0.911 & $2.96(1.07-7.40)^{a}$ & 0.020 & $2.37(1.01-5.57)^{\mathrm{a}}$ & 0.047 & $0.60(0.14-2.48)$ & 0.480 \\
\hline \multirow[t]{3}{*}{ Abdominal obesity } & I & $0.80(0.32-2.04)$ & 0.647 & $1.13(0.60-2.12)$ & 0.699 & $1.05(0.58-1.91)$ & 0.861 & $0.75(0.32-1.79)$ & 0.521 \\
\hline & II & $1.61(0.28-9.36)$ & 0.593 & $0.91(0.34-2.43)$ & 0.846 & $1.02(0.40-2.62)$ & 0.961 & $1.41(0.26-7.72)$ & 0.695 \\
\hline & III & $1.21(0.11-12.77)$ & 0.874 & $1.84(0.45-7.57)$ & 0.398 & $1.59(0.43-5.09)$ & 0.483 & $0.50(0.07-3.78)$ & 0.506 \\
\hline \multirow[t]{3}{*}{ Elevated BP } & I & $0.71(0.26-1.94)$ & 0.503 & $0.89(0.45-1.78)$ & 0.744 & $0.85(0.44-1.64)$ & 0.627 & $0.75(0.30-1.92)$ & 0.554 \\
\hline & II & $0.60(0.20-1.77)$ & 0.356 & $1.88(0.64-5.52)^{b}$ & 0.251 & $2.38(0.84-6.77)^{b}$ & 0.103 & $0.61(0.23-1.64)$ & 0.332 \\
\hline & III & $0.44(0.13-1.49)$ & 0.187 & $1.86(0.63-7.04)^{b}$ & 0.265 & $2.42(0.83-7.04)^{b}$ & 0.104 & $0.62(0.22-1.72)$ & 0.360 \\
\hline \multirow{3}{*}{$\begin{array}{l}\text { Increased glucose } \\
\text { concentration }\end{array}$} & I & $0.80(0.31-2.06)$ & 0.648 & $1.14(0.63-2.05)$ & 0.663 & $1.07(0.61-1.87)$ & 0.823 & $0.75(0.41-1.80)$ & 0.517 \\
\hline & II & $1.02(0.24-4.33)$ & 0.978 & $0.41(0.60-3.30)$ & 0.424 & $1.30(0.58-2.91)$ & 0.527 & $0.76(0.19-2.97)$ & 0.696 \\
\hline & III & $0.78(0.17-3.58)$ & 0.748 & $1.46(0.61-3.47)$ & 0.395 & $1.29(0.56(2.96)$ & 0.544 & $0.65(0.16-2.62)$ & 0.546 \\
\hline \multirow{3}{*}{$\begin{array}{l}\text { Decreased HDL } \\
\text { concentration }\end{array}$} & I & $0.56(0.19-1.64)$ & 0.290 & $1.06(0.57-1.96)$ & 0.852 & $0.95(0.52-1.172)$ & 0.861 & $0.54(0.19-1.50)$ & 0.238 \\
\hline & II & $1.44(0.31-4.78)$ & 0.642 & $1.84(0.74-4.60)$ & 0.189 & $1.72(0.72-4.11)$ & 0.219 & $1.10(0.26-4.67)$ & 0.899 \\
\hline & III & $1.26(0.25-6.21)$ & 0.778 & $1.91(0.76-4.79)$ & 0.170 & $1.72(0.71-4.16)$ & 0.224 & $0.99(0.22-4.36)$ & 0.990 \\
\hline \multirow{3}{*}{$\begin{array}{l}\text { Increased TG } \\
\text { concentration }\end{array}$} & I & $0.60(0.22-1.66)$ & 0.324 & $1.42(0.79-2.56)$ & 0.245 & $1.22(0.69-2.15)$ & 0.493 & $0.49(0.19-1.28)$ & 0.148 \\
\hline & II & $2.34(0.57-9.64)^{c}$ & 0.237 & $2.02(0.85-4.78)$ & 0.110 & $2.09(0.92-4.75)$ & 0.076 & $1.66(0.44-6.24)^{\circ}$ & 0.456 \\
\hline & III & $1.92(0.42-8.09)^{\mathrm{c}}$ & 0.399 & $2.12(0.88-5.11)$ & 0.095 & $2.15(0.93-5.00)$ & 0.075 & $1.49(0.37-5.97)^{\mathrm{c}}$ & 0.569 \\
\hline
\end{tabular}

MetS: metabolic syndrome; OR (95\% CI): odds ratio ( $95 \%$ confidence interval); BMI: body mass index; BP: blood pressure;

HDL: high density lipoprotein; TG: triglycerides.

${ }^{a}$ Statistically significant interaction gene $\times$ walking, in relation to MetS.

${ }^{b}$ Statistically significant interaction gene $\times$ walking, in relation to blood pressure.

" Statistically significant interaction gene $\times$ walking, in relation to $\mathrm{TG}$ concentration. 


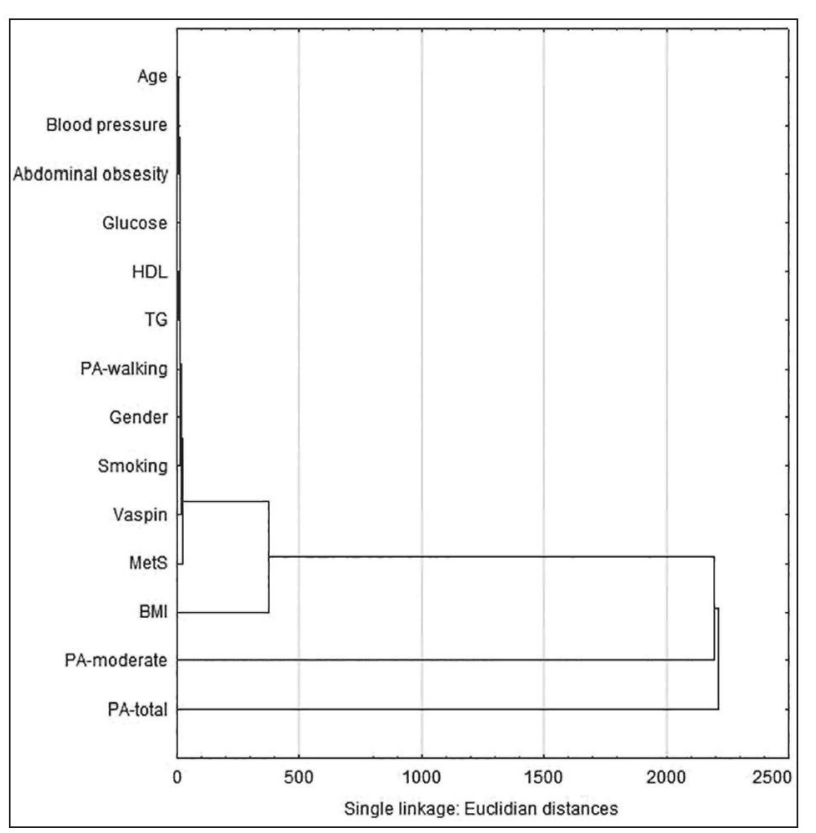

Figure 2. Schematic diagram for 14 variables; linkage distances.

MetS in the TA genotype carriers ( $\mathrm{OR}=0.24 ; p=0.022)$ and TA+AA (OR $=0.25 ; p=0.016)$, as well as the risk of elevated blood pressure in the TA genotype carriers (OR $=0.17 ; p=0.039$ and $\mathrm{TA}+\mathrm{AA}(\mathrm{OR}=0.11 ; p=0.007)$, compared to the TT carriers. It was also found that a longer walking time decreased the risk of higher TG concentration in the AA genotype carriers $(\mathrm{OR}=0.04 ; p=0.017)$, compared to TT and the AA genotype carriers, compared to $\mathrm{TT}+\mathrm{TA}(\mathrm{OR}=0.60 ; p=0.025)$.

Including BMI in the model did not significantly change the obtained scores (model III). Similar to the previous model, it was observed that longer walking time ( $>60 \mathrm{~min} . /$ day) significantly decreased the risk of MetS in $\mathrm{TA}$ and TA+AA genotype carriers $(\mathrm{OR}=0.16 ; p=0.007$ and $\mathrm{OR}=0.17 ; p=0.007$, respectively) as well as elevated blood pressure $(\mathrm{OR}=0.09 ; p=0.012$ and $\mathrm{OR}=0.05 ; p=$ 0.002 for TA and TA+AA genotypes, respectively) as well as the risk of increased TG concentration in AA genotype carriers, compared to TT (OR $=0.06 ; p=0.038)$ and AA genotype, compared to $\mathrm{TT}+\mathrm{TA}(\mathrm{OR}=0.07 ; p=0.043)$.

\section{DISCUSSION}

In our study, the possible association between the vaspin rs2236242 gene polymorphism and the risk of developing MetS in a group of Polish men and women was investigated. To the best of our knowledge, this is the first time such an association has been studied in the Polish population. Both in the unadjusted and adjusted models, there have been no statistically significant associa- tion between the vaspin rs 2236242 polymorphism and the risk of the occurrence of individual components of MetS. However, in the model adjusted for known confounders (smoking, BMI, walking), it was noted that carrying the TA genotype was related to a greater risk of MetS, compared to the TT genotype.

Results of other studies are ambiguous in this respect. Hashemi et al. [7] in a study conducted on Iranian population observed that the A allele of the vaspin rs 2236242 polymorphism plays a protective role against MetS. The risk of MetS was decreased significantly by being a carrier of the AA and TA genotypes compared to the TT genotype. The associations remained almost unchanged after adjusted for gender and age [7]. Mehanna et al. [8] in a study of Egyptian women, also found that the rare A allele of the vaspin rs2236242 polymorphism and the TA and AA genotypes appeared with higher frequencies among the control group individuals than in MetS subjects. A similar tendency, related to the protective function of the AA genotype against MetS, was shown in the results of our study, in the unadjusted model. The tendency to a slightly more frequent occurrence of TA genotype in MetS-subjects, compared to control-subjects (51.86 vs. $45.45 \%$ ), as well as a higher risk of MetS in TA genotype carriers. However, these results did not reach a level of statistical significance. Different results were achieved on Egyptian women by Alnory et al. [6] who did not observe any significant associations between the $A$ allele of the vaspin rs2236242 polymorphism and risk of MetS when compared to the $\mathrm{T}$ allele. The $\mathrm{AA}$ and $\mathrm{AT}$ genotypes also showed no association with MetS when compared to the TT genotype. Moreover, the authors did not find any associations between genotypes of the vaspin rs 2236242 polymorphism and different serum vaspin levels [6].

In the Iranian population, the analysis of the associations between the vaspin rs 2236242 gene polymorphism and health conditions such as obesity, PCOS and T2DM, showed ambiguous results. Kohan et al. [11] observed a decreased risk of PCOS in the A allele carriers when compared to the T carriers. However, this relationship was not statistically significant after adjusting genotypes for BMI [12]. Abdel Ghany et al. [12] concluded that the minor (A) allele of the vaspin rs2236242 gene polymorphism occurred less frequently in obese women compared to control group. It played a protective role against obesity in dominant, codominant, additive, and recessive inheritance models. However, after adjusting genotypes for T2DM there were no significant associations between polymorphism of this gene and obesity. According to the authors, the vaspin rs 2236242 gene polymorphism shows protective effects in obesity, but this association results mainly from its effect on insulin sensitivity [12]. The 
analysis of the German population conducted by Kempf et al. [13] revealed that the AA genotype carriers had an increased risk of T2DM, compared with the TT genotype carriers, but contrary to all other studies they analyze the vaspin rs 2236242 polymorphism in the reverse strand of the vaspin gene. The results of the meta-analysis of three populations only enforce the theory that A allele plays a protective role against MetS occurrence and TT genotype increase the risk of syndrome progression.

It has been shown that lifestyle factors, such as physical activity, can modify associations between variants of several genes and the risk of developing obesity, T2DM and cardiovascular disease in various populations [14-16]. Metabolic syndrome is also treated as a result of interactions between genetic factors and unhealthy lifestyle. It is thought that despite the significant role of genetic factors, a lack of regular PA and obesity are the main causes of the increase of occurrence of metabolic disorders, especially in developed countries $[17,18]$. According to our current knowledge, none of the previously published studies on the relationship between the vaspin rs 2236242 polymorphism and the risk of MetS included PA or walking. Thus, our study provides the first vaspin rs2236242 gene polymorphism $\times \mathrm{PA} /$ walking time interaction data on MetS and its components. Studies of the influence of other genes and PA on adiposity and metabolic characteristics provide diverse results. Brito et al. [19] evaluated the effect of 'genes-physical activity' interaction on impaired glucose regulation risk for 17 loci related to T2DM and showed that three polymorphisms were statistically significant: CDKN2A/B rs 10811661, HNF1B rs4430796 and PPARG rs 1801282. Similar to our analysis, the inclusion or exclusion of BMI as a covariate in the SNP $\times$ physical activity interaction models made no essential difference to the interaction results. Thus the authors concluded that the genetic predisposition to hyperglycemia is partially dependent on a person's lifestyle [19]. Kilpeläinen [14] found that the effect of increasing of BMI by rs 9939609 gene FTO polymorphism was weakened by $27.0 \%$ in physically active individuals compared to non active ones. However, Graff et al. [20] did not find any evidence of the interaction with PA for loci other than FTO.

The analysis of interactions revealed that in our study population, longer walking ( $>60 \mathrm{~min}$./day) lowered the risk of MetS and elevated blood pressure in the TA and TA+ AA genotype carriers and the risk of increased TG concentration in the AA genotype carriers, results were statistically significant. Walking is the most frequent form of physical activity. Results of several studies show that despite its low intensity, it is associated with lower risks of cardiovascular disease, T2DM and all cause mortality in men and women $[21,22]$. In a prospective study of
Japanese workers aged 30-69, walking less than $60 \mathrm{~min} . /$ day comprised one of the crucial lifestyle elements increasing the risk of MetS, during 1 year of observation [23]. A decrease of MetS occurrence related to longer walking time, was also observed in several cross-sectional studies [24] and meta-analysis of cohort studies [25].

The strength of the conducted study is the inclusion of confounding factors in statistical analysis, which may have a modifying effect on the risk of MetS occurrence and its components. The limitations are considered to be the small number of participants and the fact that PA was assessed with the use of questionnaires, which are prone to bias and errors.

Conclusions. In the unadjusted model, no associations between the vaspin rs2236242 polymorphism and the risk of MetS and its components have been observed. Only the tendency to a decreased risk of MetS and its components in the AA genotype carriers has been noted, but the result was statistically insignificant. The results of the conducted study suggest that any unfavorable effect of the TA genotype of the vaspin rs 2236242 polymorphism can be essentially reduced, or even reversed, in a case of individuals walking longer than $60 \mathrm{~min}$. The analysis of interaction between the vaspin rs2236242 gene polymorphism and walking has revealed that walking time longer than $60 \mathrm{~min}$./day considerably reduces the risk of MetS, elevated blood pressure and TG concentration. It is necessary to conduct further studies on a bigger group of participants, which will allow us to determine whether these results will be useful in MetS prophylaxis.

Declaration of Interest. The authors report no conflicts of interest. The authors alone are responsible for the content and writing of this article.

Funding. This study was conducted with the support of the Maria Skłodowska-Curie Institute of Oncology in Warsaw and the Polish-Norwegian Foundation Research Fund. The research data were collected within the scope of PONS research: "Establishing infrastructure for studies concerning health state of the population of Poland" (PNRF-228-AI-1/07) (data collection). This study was supported by a grant from The Ministry of Science and Higher Education from the funds received within financing statutory activity for Faculty of Medicine and Health Sciences, Jan Kochanowski University, research project No. 615507 (data analysis and preparation of the manuscript). This study was supported under the program of the Minister of Science and Higher Education under the name Regional Initiative of Excellence in 2019-2022 project number: 024/RID/2018/19, financing amount: 11.999.000,00 PLN. 


\section{REFERENCES}

1. Suliga E, Wawszczak M, Gluszek S. The roles of vaspin, chemerin, and omentin in the determination of metabolic syndrome. Med Studies. 2018; 34(2): 160-177.

2. Feng $\mathrm{R}, \mathrm{Li} Y$, Wang $\mathrm{C}$, Luo $\mathrm{C}$, Liu L. Higher vaspin levels in subjects with obesity and type 2 diabetes mellitus: A meta-analysis. Diabetes Res Clin Pract. 2014; 106(1): 88-94.

3. Auguet T, Quintero Y, Riesco D, Morancho B, Terra $\mathrm{X}$, Crescenti $\mathrm{A}$, et al. New adipokines vaspin and omentin. Circulating levels and gene expression in adipose tissue from morbidly obese women. BMC Med Genet. 2011; 12: 60.

4. Mahdirejei HA, Abadei SFR, Seidi AA, Eshaghei Gorji N, Rahmani Kafshgari H, Ebrahim Pour M, et al. Effects of an eight-week resistance training on plasma vaspin concentrations, metabolic parameters levels and physical fitness in patients with type 2 diabetes. Cell J. 2014; 16(3): 367-374.

5. Esteghamati A, Noshad S, Mousavizadeh M, Zandieh A, Nakhjavani M. Association of vaspin with metabolic syndrome: The pivotal role of insulin resistance. Diabetes Metabol J. 2014; 38(2): 143-149.

6. Alnory A, Gad H, Hegazy G, Shaker O. The association of vaspin rs2236242 and leptin rs7799039 polymorphism with metabolic syndrome in Egyptian women. Turkish J Med Sci. 2016; 46(5):1335-1340.

7. Hashemi M, Rezaei H, Eskandari-Nasab E, Kaykhaei MA, Zakeri Z, Taheri M. Association between chemerin rs17173608 and vaspin rs2236242 gene poly-morphisms and the metabolic syndrome, a preliminary report. Gene. 2012; 510(2): 113-117.

8. Mehanna ET, Mesbah NM, Ghattas MH, Saleh SM, Abo-Elmatty DM. Association of chemerin Rs17173608 and vaspin Rs2236242 gene polymorphisms with metabolic syndrome in Egyptian women. Endocr Res. 2016; 41(1): 43-48.

9. Suliga E, Kozieł D, Cieśla E, Rębak D, Głuszek S. Dietary patterns in relation to metabolic syndrome among adults in Poland: A cross-sectional study. $\mathrm{Nu}-$ trients. 2017; 9(12): E1366.

10. Alberti KGMM, Eckel RH, Grundy SM, Zimmet PZ, Cleeman JI, Donato KA, et al. Harmonizing the metabolic syndrome: A joint interim statement of the International Diabetes Federation Task Force on Epidemiology and Prevention; National Heart, Lung, and Blood Institute; American Heart Association; World Heart Federation; International Atheroscle- rosis Society; and International Association for the Study of Obesity. Circulation. 2009; 120(16):16401645.

11. Kohan L, Zarei A, Fallahi S, Tabiee O. Association between vaspin rs2236242 gene polymorphism and polycystic ovary syndrome risk. Gene. 2014; 539(2): 209-212.

12. Abdel Ghany SM, Sayed AA, El-deek SEM, Elbadre HM, Dahpy MA, Saleh MA, et al. Obesity risk prediction among women of Upper Egypt: The impact of serum vaspin and vaspin rs2236242 gene polymorphism. Gene. 2017; 626(1): 140-148.

13. KempfK, Rose B, Illig T, Rathmann W, Strassburger $\mathrm{K}$, Thorand B, et al. Vaspin (SERPINA12) genotypes and risk of type 2 diabetes: Results from the MONICA/ KORA studies. Exp Clin Endocrinol Diabetes. 2008; 118(3): 184-189.

14. Kilpeläinen TO, Qi L, Brage S, Sharp SJ, Sone-stedt $\mathrm{E}$, Demerath E, et al. Physical activity attenuates the influence of FTO variants on obesity risk: A metaanalysis of 218,166 adults and 19,268 children. PLoS Med. 2011; 8(11): e1001116.

15. Kilpeläinen TO, Franks $P W$. Gene-physical activity interactions and their impact on diabetes. Diabetes Phys Act. 2014; 60: 94-103.

16. Abdullah MS, Verweij N, Van Der Harst P. Associations of combined genetic and lifestyle risks with incident cardiovascular disease and diabetes in the UK biobank study. JAMA Cardiol. 2018; 3(8): 693 702 .

17. Grundy SM, Cleeman J, Daniels SR, Donato KA, Eckel RH, Franklin BA, et al. Diagnosis and management of the metabolic syndrome. Circulation. 2005; 112(17): 2735-2752.

18. Paley CA, Johnson MI. Abdominal obesity and metabolic syndrome: exercise as medicine? BMC Sports Sci Med Rehabil. 2018; 10: 7.

19. Brito EC, Lyssenko V, Renstrom F, Berglund G, Nilsson PM, Groop L, et al. Previously associated type 2 diabetes variants may interact with physical activity to modify the risk of impaired glucose regulation and type 2 diabetes: A study of 16,003 Swedish adults. Diabetes. 2009; 58(6): 1411-1418.

20. Graff M, Scott RA, Justice AE, Young KL, Feitosa MF, Barata L, et al. Genome-wide physical activity interactions in adiposity -A meta-analysis of 200,452 adults. PLoS Genet. 2017; 13(4): e1006528.

21. Hamer M, Chida Y.Walking and primary prevention: A meta-analysis of prospective cohort studies. $\mathrm{Br} \mathrm{J}$ Sports Med. 2008; 42(4): 238-243. 
22. Kato M, Goto A, Tanaka T, Sasaki S, Igata A, Noda M. Effects of walking on medical cost: A quantitative evaluation by simulation focusing on diabetes. J Diabetes Invest. 2013; 4(6): 667-672.

23. Tajima M, Lee JS, Watanabe E, Park JS, Tsuchiya $\mathrm{R}$, Fukahori A, et al. Association between changes in 12 lifestyle behaviors and the development of metabolic syndrome during 1 year among workers in the Tokyo Metropolitan area. Circ J. 2014; 78(5): 1152-1159.
24. Kim SH, Lee SH, Ahn KY, Lee DH, Suh YJ, Cho $\mathrm{SG}$, et al. Effect of lifestyle modification on serum chemerin concentration and its association with insulin sensitivity in overweight and obese adults with type 2 diabetes. Clin Endocrinol. 2014; 80(6): 825-833.

25. Zhang D, Liu X, Liu Y, Sun X, Wang B, Ren Y, et al. Leisure-time physical activity and incident metabolic syndrome: A systematic review and dose-response meta-analysis of cohort studies. Metabolism. 2017; 75: 36-44. 